\title{
Long-term developmental follow-up in children with nonsyndromic craniosynostosis
}

\author{
Maggie Bellew, PhD, ${ }^{1}$ and Paul Chumas, FRCS(SN)² \\ Departments of ${ }^{1}$ Plastic, Reconstructive, and Hand Surgery and ${ }^{2}$ Neurosurgery, Leeds General Infirmary, Leeds, United Kingdom
}

OBJECT The aim of this study was to determine the distribution of Full Scale IQ (FSIQ) by type of craniosynostosis and to verify the finding that at long-term follow-up, verbal IQ $(\mathrm{VIQ})$ is significantly higher than performance IQ (PIQ) in patients with single-suture sagittal synostosis (SS) despite falling within the "average" range for intelligence. Whether this also occurs in other types of craniosynostosis and whether surgery and sex are relevant were also determined. The relationship between age at time of surgery and later $I Q$ was ascertained.

METHODS The data for 91 children with craniosynostosis (47 sagittal, 15 unicoronal, 13 metopic, 9 multisuture, and 7 bicoronal) were collected at their routine, 10 years of age IQ assessment (mean age 123.8 months). The patients included 61 males and 30 females; 62 patients had undergone surgery and 29 had not.

RESULTS The mean FSIQ for all types of craniosynostosis combined (96.2) fell within the average range for the general population. Some variation was evident across the different types of craniosynostosis: the SS group showed the highest FSIQs and a "normal" distribution of bandings; the other types had a higher proportion of FSIQs in the lower bandings. The data confirmed the finding that $V I Q$ is greater than PIQ despite falling within the average range for intelligence, with a difference of 5.0 for all types of craniosynostosis combined ( $p=0.001), 7.6$ for the SS group $(p=0.001)$, and 6.9 for the unicoronal group $(p=0.029)$. This $V I Q>P I Q$ effect was not found with multisuture craniosynostosis. The $V I Q>P I Q$ discrepancy occurred regardless of whether the patient had undergone surgery and occurred more often in males than females. In the SS group and the bicoronal group, FSIQ ( $p=0.036$ and $p=0.046$, respectively) and PIQ ( $p=$ 0.012 and $p=0.017$, respectively), though not $\mathrm{VIQ}$, were higher when surgery had been performed early.

CONCLUSIONS The study confirms that at long-term follow-up, although children with nonsyndromic craniosynostosis fall within the normal range for intelligence, there is a VIQ $>$ PIQ discrepancy above what would be expected in the normal population, which may be indicative of more subtle difficulties in achievement. This discrepancy is affected by type of craniosynostosis, sex, and age at time of surgery.

http://thejns.org/doi/abs/10.3171/2015.3.PEDS14567

KEY WORDS craniosynostosis; long-term follow up; developmental attainment; IQ; craniofacial surgery; children

I N 2002, Magge et al. ${ }^{16}$ found that with a small-sample study of 16 patients of varied ages (6.4-15.9 years, mean 10.3 years) with nonsyndromic single-suture sagittal synostosis (SS), verbal IQ (VIQ) was statistically significantly higher $(\mathrm{p}=0.021)$ than performance IQ (PIQ), as measured by the Wechsler Intelligence Scale for Children 3rd edition (WISC-III). ${ }^{25}$ This was despite falling within the "average" range for intelligence. The current study set out to see if these results could be replicated with a larger sample of more uniform age and with other types of craniosynostosis included. The roles of surgery and sex were also examined. There is a paucity of information on long-term follow-up in craniosynostosis, which this study goes some way to address.

\section{Methods}

The Leeds (East) Research Ethics Committee confirmed that "ethics committee approval is not needed for the collation and analysis of patient data collected over a period of time on a routine basis during normal clinical care, on the understanding that the data is pooled and therefore nonidentifiable."

All children attending the Leeds Regional craniofacial center are invited for regular developmental review

ABBREVIATIONS FSIQ = Full Scale IQ; ICP = intracranial pressure; PIQ = performance IQ; $S S=$ sagittal synostosis; VIQ = verbal IQ; WASI = Wechsler Abbreviated Scale of Intelligence; WISC = Wechsler Intelligence Scale for Children

SUBMITTED October 15, 2014. ACCEPTED March 23, 2015.

INCLUDE WHEN CITING Published online July 24, 2015; DOI: 10.3171/2015.3.PEDS14567.

DISCLOSURE The authors report no conflict of interest concerning the materials or methods used in this study or the findings specified in this paper. 
according to a standardized protocol: preoperatively; 6 months postoperatively if receiving surgery (or at a similar interval if not receiving surgery); and at 4, 10, and 15 years of age. Additional assessments may be conducted according to clinical need.

The data from 91 consecutive patients with craniosynostosis who had attended their routine 10 years of age developmental assessment at the Leeds Craniofacial Unit were collated, from the first patient to reach 10 years of age (February 2003) until January 2014. One patient underwent early assessment, at 8.02 years, due to clinical requirements, and 4 patients attended just prior to their tenth birthdays. Eight patients attended at 11 years of age due to difficulties in attending appointments. A further 81 patients did not attend their 10-year review or had been lost to follow-up (SS $46.9 \%$, metopic $24.6 \%$, unicoronal $11.1 \%$, bicoronal $3.7 \%$, multi/other $13.6 \%$ ).

All the assessments were carried out by clinical psychologists who were blinded to the VIQ > PIQ hypothesis and had not read the Magge et al. ${ }^{16}$ paper. Table 1 presents the number of patients with each type of craniosynostosis along with their sexes and their mean age at the time of assessment. The table also shows whether patients had undergone surgery or not and their ages at the time of surgery. The mothers of 2 children (1 with metopic craniosynostosis, 1 with SS) had taken sodium valproate during pregnancy.

Plain radiographs were obtained to confirm the clinical diagnosis of craniosynostosis. CT scanning or MRI were also performed in cases of metopic synostosis (to look for associated brain malformations) or in more complex multisuture cases. Children who did not undergo surgery usually had cosmetically mild craniosynostosis. Those patients with more marked disease whose parents did not wish them to undergo surgery were closely observed clinically (ophthalmic and developmental review and serial head circumference measurements), with a low threshold for intracranial pressure (ICP) monitoring if there was any concern. Patients under 6 months of age with SS underwent a modified Renier's " $H$ " procedure. ${ }^{8}$ Older patients ( $>6$ months and usually around 1 year of age) with significant occipital bullets or frontal bossing had these aspects corrected in addition to undergoing the basic Renier technique. Patients with metopic, unicoronal, or bicoronal types were treated by frontal advancement (using either a Marchac frontal template or reversal of the frontal bone).
All the patients were referred for fundus examination by an expert pediatric ophthalmologist.

All patients had been assessed using the WISC 4th edition, UK edition (WISC-IV UK) ${ }^{26}$ or the shorter version of this scale, The Wechsler Abbreviated Scale of Intelligence (WASI), ${ }^{24}$ which correlates well with the WISCIV UK. Both are individually administered, standardized measures of intelligence, and they yield Full Scale IQ (FSIQ), verbal IQ (VIQ) and performance IQ (PIQ). The FSIQ is the overall estimate of an individual's general level of intellectual functioning. The VIQ is a measure of acquired knowledge, verbal reasoning, and attention to verbal information. The PIQ provides an indication of nonverbal skills and is a measure of fluid reasoning, spatial processing, attentiveness to detail, and visual-motor integration.

Statistical analyses were conducted using SPSS version 19.0 for Windows (IBM). Independent sample t-tests were undertaken to compare FSIQs between different types of craniosynostosis, and the distribution of FSIQs within types was also examined. Paired t-tests were performed to compare VIQ with PIQ. This was undertaken for all types of craniosynostosis combined, and then for each type separately. The data were also analyzed separately for surgical and nonsurgical patients and for male and female patients. One-way ANOVAs were used to examine whether age at time of surgery was relevant.

\section{Results \\ FSIQ Data}

Table 2 shows that for all types of craniosynostosis combined, the mean FSIQ (96.2) fell within the average range for the child population. There was some variation across the different types of craniosynostosis, with the SS group showing the highest mean FSIQ for simple craniosynostosis (100.5). The other types all had significantly lower mean FSIQs than the SS group, falling within the "low average" range of ability: unicoronal $(\mathrm{p}=0.036)$, metopic $(\mathrm{p}=0.043)$, and bicoronal $(\mathrm{p}=0.039)$.

Table 3 shows that the distribution of FSIQs varied according to type of craniosynostosis, with the SS group having the most "normal" distribution and most similar to the published WASI norms. The other types had a higher proportion of FSIQs in the "borderline learning difficulties" or "learning difficulties" range of ability compared

TABLE 1. Type of craniosynostosis, sex, age at time of surgery, and age at time of developmental assessment

\begin{tabular}{|c|c|c|c|c|c|c|c|c|c|}
\hline \multirow[b]{2}{*}{ Type of Craniosynostosis } & \multirow[b]{2}{*}{ No. of Pts } & \multirow[b]{2}{*}{$\mathrm{M}, \mathrm{F}$} & \multirow[b]{2}{*}{ Surgery } & \multirow[b]{2}{*}{ No Surgery } & \multicolumn{3}{|c|}{ Age at Time of Surgery* } & \multicolumn{2}{|c|}{ Age at Time of Assessment* } \\
\hline & & & & & $<7$ & $7-18$ & $>19$ & Mean (SD) & Range \\
\hline Sagittal & 47 & 39,8 & 33 & 14 & 14 & 14 & 5 & $123.1(6.57)$ & $96.2-138.6$ \\
\hline Unicoronal & 15 & 5,10 & 11 & 4 & 1 & 9 & 1 & $124.2(3.81)$ & $120.6-132.0$ \\
\hline Metopic & 13 & 9,4 & 5 & 8 & 1 & 4 & 0 & $124.3(5.79)$ & $120.1-142.0$ \\
\hline Bicoronal & 7 & 4,3 & 6 & 1 & 0 & 5 & 1 & $127.3(7.36)$ & $122.2-142.5$ \\
\hline Multisuture & 9 & 4,5 & 7 & 2 & 1 & 4 & 2 & $122.8(2.63)$ & $120.2-128.4$ \\
\hline Total & 91 & 61,30 & 62 & 29 & 17 & 36 & 9 & $123.8(5.87)$ & $96.2-142.5$ \\
\hline
\end{tabular}

Pts = patients.

* Values are months. 
with the SS group, with very few children scoring in the higher bandings.

There was no significant difference when male patients were compared with female patients in terms of FSIQ data, either for all types of craniosynostosis combined or for each type separately.

\section{VIQ and PIQ Data}

Impact of Diagnosis

The data also confirm Magge et al.'s ${ }^{16}$ VIQ $>$ PIQ finding for SS craniosynostosis (Table 2). In our study, there was a difference of 5.0 for all types of craniosynostosis combined $(\mathrm{p}=0.001), 7.6$ for the SS group $(\mathrm{p}=0.001)$, and 6.9 for the unicoronal group $(\mathrm{p}=0.029)$.

VIQ $\geq$ PIQ only appeared in the single-suture groups. There was a trend toward it being the other way round (i.e., PIQ > VIQ) in the bicoronal and combined multisuture synostosis groups.

\section{Impact of Surgery}

Further analysis suggested that a statistically significant effect only occurred in surgical patients $(p=0.001$ for all types of craniosynostosis combined, $p=0.002$ for the SS group, $p=0.026$ for the unicoronal group). This may, of course, reflect condition severity rather than whether surgery had taken place, surgery being less likely to be undertaken in milder cases. However, VIQ > PIQ was found in $22(66.7 \%)$ of the 33 cases in the SS surgical group and in $10(71.4 \%)$ of the 14 cases in the SS nonsurgical group. The lack of statistical significance may, therefore, just reflect small sample size.

\section{Impact of Sex}

Table 4 shows that VIQ > PIQ only seemed to occur within groups of male patients, with a difference of 6.5 for all types of craniosynostosis combined $(p=0.0001)$ and a difference of 7.9 for the SS group male patients $(\mathrm{p}=$ 0.001 ). While $72 \%$ of the male SS patients demonstrated VIQ > PIQ, only $50 \%$ of female SS patients showed this IQ discrepancy (68.1\% for male and female combined). There were no significant effects for the female groups (though it nearly reached significance for the unicoronal group, with $90 \%$ of females showing VIQ > PIQ compared with $60 \%$ of males). Furthermore, while VIQ > PIQ was always the case for the male groups, it was the other way round for the metopic, bicoronal, and multisuture females, with the majority of these female patients demonstrating PIQ > VIQ. Overall, $67 \%$ of males showed VIQ > PIQ, but only $53 \%$ of females showed this discrepancy.

When male patients were compared directly with female patients in terms of their actual VIQ or PIQ (rather than the difference between VIQ and PIQ), either for all types of craniosynostosis combined or for each type separately, there were no significant differences.

\section{Age at Time of Surgery}

The data were also examined to establish whether age at time of surgery was relevant. This was first determined for all types of craniosynostosis combined. In terms of FSIQ, PIQ, and VIQ, 1-way ANOVAs comparing surgery

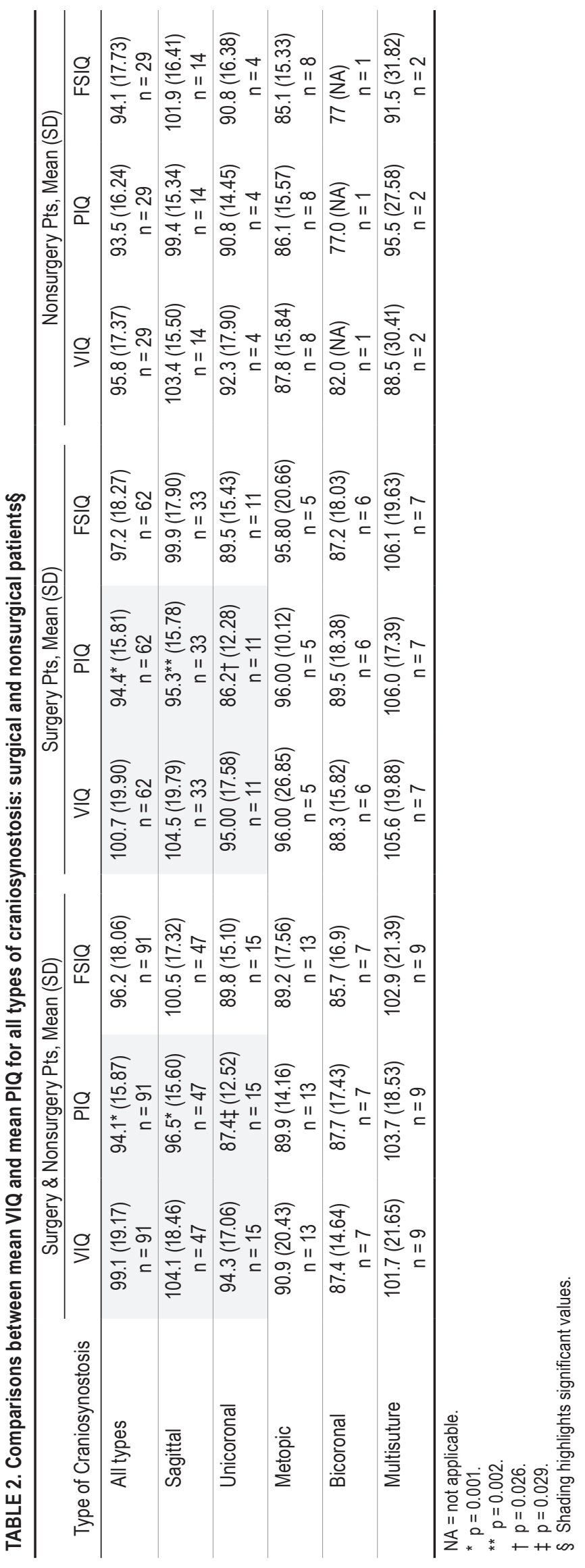

J Neurosurg Pediatr Volume 16 • October 2015 
TABLE 3. Distribution of FSIQ for all types of craniosynostosis and comparison with WASI norms*

\begin{tabular}{|c|c|c|c|c|c|c|c|}
\hline $\begin{array}{c}\text { Type of } \\
\text { Craniosynostosis }\end{array}$ & $\begin{array}{l}\text { Very Superior } \\
\quad \geq 130 \\
(\text { Norm }=2.0 \%)\end{array}$ & $\begin{array}{c}\text { Superior } \\
120-129 \\
(\text { Norm }=7.3 \%)\end{array}$ & $\begin{array}{c}\text { High Average } \\
110-119 \\
(\text { Norm }=15.6 \%)\end{array}$ & $\begin{array}{c}\text { Average } \\
90-109 \\
(\text { Norm }=50.0 \%)\end{array}$ & $\begin{array}{c}\text { Low Average } \\
80-89 \\
\text { (Norm }=15.8 \%)\end{array}$ & $\begin{array}{c}\text { Borderline } \\
70-79 \\
(\text { Norm }=6.8 \%)\end{array}$ & $\begin{array}{c}\text { Extremely Low } \\
\quad \leq 69 \\
(\text { Norm }=2.5 \%)\end{array}$ \\
\hline Sagittal, $n=47$ & $2(4.3)$ & $3(6.4)$ & $11(23.4)$ & $18(38.3)$ & $8(17)$ & $4(8.5)$ & $1(2)$ \\
\hline Unicoronal, $n=15$ & 0 & 0 & $2(13.3)$ & $7(46.7)$ & $2(13.3)$ & $2(13.3)$ & $2(13.3)$ \\
\hline Metopic, $n=13$ & 0 & $1(7.7)$ & 0 & $5(38.5)$ & $3(23.1)$ & $3(23.1)$ & $1(7.7)$ \\
\hline Bicoronal, $n=7$ & 0 & 0 & 0 & $2(28.6)$ & $2(28.6)$ & $2(28.6)$ & $1(14.3)$ \\
\hline Multisuture, $n=9$ & $1(11.1)$ & $1(11.1)$ & $2(22.2)$ & $2(22.2)$ & $2(22.2)$ & - & $1(11.1)$ \\
\hline Total, $n=91$ & $3(3.3)$ & $5(5.5)$ & $15(16.5)$ & $34(37.4)$ & $17(18.7)$ & $11(12.1)$ & $6(6.6)$ \\
\hline
\end{tabular}

* Figures in parentheses are the percentage of patients falling within that banding.

performed at $\leq 6$ months of age with that performed at $7-18$ months of age and $\geq 19$ months showed no significant results.

There were, however, significant results when patient FSIQs were examined by type of craniosynostosis. Oneway ANOVAs showed a significant difference in FSIQ for SS patients $(p=0.036)$, with the highest mean FSIQ being obtained when surgery was performed at $\leq 6$ months of age (FSIQ 108.7), followed by surgery at 7-18 months (FSIQ 95.2), and lowest when surgery was performed at $\geq 19$ months (FSIQ 88.6). There were similar results for patients with bicoronal synostosis $(\mathrm{p}=0.046)$, with those patients undergoing surgery at 7-18 months obtaining a higher mean FSIQ (93.2) compared with those undergoing surgery at $\geq 19$ months (FSIQ 57.0). The number of patients with bicoronal synostosis was, however, very small, and no one in this group had undergone surgery at $\leq 6$ months of age.

One-way ANOVAs for PIQ across the surgery age groups of $\leq 6,7-18$, and $\geq 19$ months were very similar, with a significant result for the SS group $(p=0.012)$ (PIQs of 104.2, 90.1, and 84.8, respectively) and for the bicoronal group ( $\mathrm{p}=0.017$ ) (PIQs of 96.2 for surgery at 7-18 months and 56.0 for surgery at $\geq 19$ months). Again, there were no significant results for VIQ.

\section{Discussion}

\section{Long-Term Developmental Outcome}

Some previous studies ${ }^{7,13,14,22,23}$ have suggested that there may be long-term developmental and educational difficulties in children with craniosynostosis, even in SS, generally considered to be the most benign of the craniosynostotic conditions. Kapp-Simon et al. ${ }^{14}$ concluded that the literature suggests that children with single-suture craniosynostosis have "mild but persistent neuropsychological deficits, which become more significant as cognitive demands increase at school age." Speltz et al. ${ }^{22}$ found that isolated craniosynostosis was associated with a 3- to 5 -fold increase in risk for cognitive deficits or learning/ language disabilities.

In a long-term follow-up of 65 adolescents (approximately 14 years of age) who had undergone surgery for SS or unicoronal craniosynostosis when they were under 12 months of age, Chieffo et al. ${ }^{7}$ found that $7 \%$ of their sample with SS demonstrated visuospatial and construc- tional ability defects with associated visual memory recall deficits; $17 \%$ also exhibited selective and sustained attention deficits. Approximately one-third (30\%) of their sample with anterior plagiocephaly had deficits in processing and planning speech. Their data, therefore, supported the hypothesis that children with SS or unicoronal craniosynostosis may still manifest lower than average results at long-term selective neuropsychological evaluations, even if they have undergone early surgical treatment.

Magge et al. ${ }^{16}$ argued that a VIQ > PIQ discrepancy can represent a risk of developing a learning disability and is suggestive of visual-motor deficiencies, and the authors go on to identify a higher incidence (50\%) of learning disabilities (reading, spelling, and visual-motor problems) in their patient group compared with the general population (5\%). This is despite the fact that their IQs fall within the normal range, with the mean PIQ being in the average range and the mean VIQ and FSIQ being in the high average range. They argued that despite their normal IQ, this is not borne out in measures of achievement or performance, which are detecting more subtle difficulties (underachievement in literacy).

The current study replicates Magge et al.'s ${ }^{16}$ finding of a VIQ > PIQ discrepancy in children with nonsyndromic craniosynostosis who fall within the normal range for intelligence (with a much larger sample size and more uniform age). However, although both studies find this discrepancy to be statistically significant when the difference in scores is analyzed for a group of patients combined, at an individual level, a difference of 8.3 in the Magge et al. ${ }^{16}$ study would only be significant at a $\mathrm{p}=0.15$ level, and the degree of difference in the current study would also not be considered statistically significant. There is also the issue that a statistically significant difference is not the same as a clinically meaningful difference. It is possible for the difference between an individual's VIQ and PIQ to be significant in the statistical sense, but for it not to be rare among the general population. For instance, 25\% of the population would show a VIQ > PIQ discrepancy of 10-points within the ability range of Magge et al.'s $\mathrm{s}^{16}$ patient population. Unfortunately, those authors did not report how many of the patient group showed the VIQ > PIQ discrepancy. However, in the current study, $68.1 \%$ of the SS group showed the VIQ > PIQ discrepancy, and this is clearly above what would be expected in the general population. 
Furthermore, in the later study by Chieffo et al., ${ }^{7}$ a difference of more than 12 points was found between VIQ (mean 111.4) and PIQ (mean 99) in SS patients. However, there was no difference in right coronal patients (mean VIQ 99.5, mean PIQ 95.6), and the difference was reversed in left coronal patients (mean VIQ 92.6, mean PIQ 105).

The current data are consistent with the literature and also with our earlier findings of specific areas of locomotor difficulty at younger ages (Bellew et al. ${ }^{4,5}$ ). These earlier data indicated that children with SS have significantly poorer gross locomotor function than the normal controls. However, following surgical intervention, the deficit was shown to have resolved and, consistent with this, a lesser improvement in eye-hand coordination and performance skills was shown. Overall developmental attainment also improved postoperatively. The children with SS who did not undergo surgery did not show any improvement in development. These findings were maintained and improved upon by 5 years of age. The current data suggest that despite this finding, verbal skills remain superior to performance skills and may indicate nonverbal learning difficulties.

This is consistent with (noncraniofacial) work by Neumann and Walker, ${ }^{17}$ who argued that neuromotor soft signs (e.g., motor coordination problems) at a young age may later persist as subtle perceptual-motor deficits in older children and adults and may be predictive of cognitive, behavioral, and psychiatric problems in later childhood and adolescence. It is not, however, consistent with previous studies that indicate that those with SS are at increased risk of speech and language impairment, particularly expressive language impairment, despite no global cognitive impairment. ${ }^{20}$

\section{IQ in Different Types of Craniosynostosis}

The current data also revealed some variation across the different types of craniosynostosis, with children in the SS group showing the highest FSIQ and the most normal distribution. This is consistent with the view that SS is usually associated with normal development and represents more of a cosmetic problem. ${ }^{1}$ Children with other types of craniosynostosis had significantly lower FSIQs, which did not show a normal distribution. (Chieffo et al. ${ }^{7}$ had a similar finding with their SS and unicoronal craniosynostosis patients.) The bicoronal synostosis group had the lowest attainment, and this is consistent with the view that developmental delay is more likely the more sutures involved..$^{12}$ This was not, however, borne out by the FSIQ of the multisuture group, although it should be noted that this group was disadvantaged in the study by having a small sample size, a range of individual diagnoses, and a large range of FSIQs (69-134). The data for the metopic patients is also consistent with the literature, which suggests that metopic craniosynostosis is associated with a higher rate of developmental delay than other types of nonsyndromic craniosynostosis. Cognitive and behavioral abnormalities have been reported in about a third of metopic patients. ${ }^{6,21}$

\section{Surgery and Developmental Outcome}

Magge et al. ${ }^{16}$ also pointed out that previous studies ${ }^{12}$

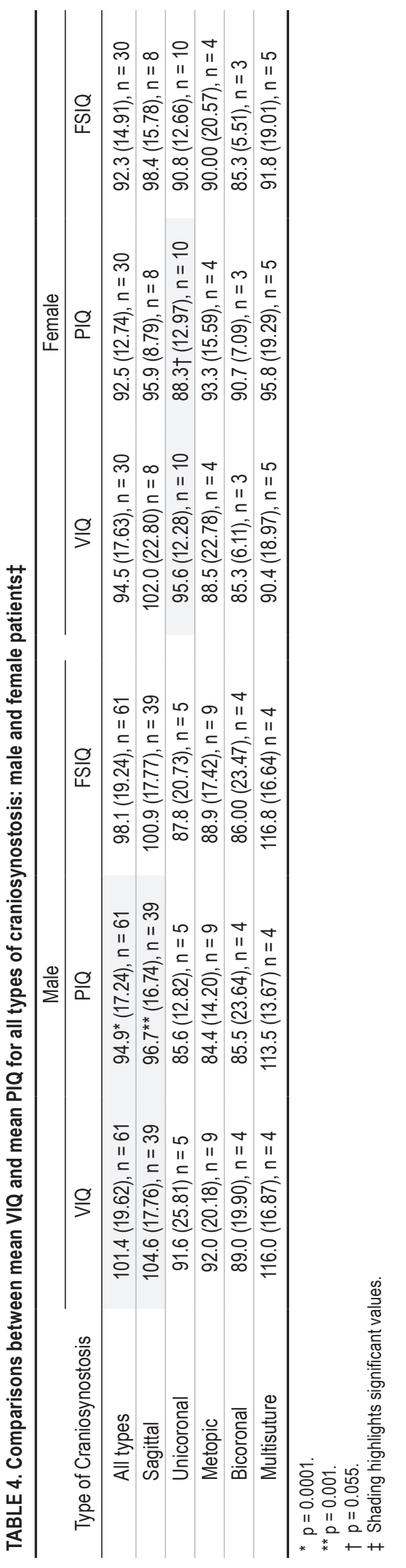

J Neurosurg Pediatr Volume 16 • October 2015 
have asserted that children operated on at a younger age (under 1 year) have better outcomes. They did not find any evidence of this in their study, but felt the issue had not been adequately explored, as none of the children in their study had undergone cranial reconstruction after 1 year of age. In our current study, the age range at time of surgery was much wider (3.1-60.3 months), with 21 children in the group overall (10 children in the SS group alone) above the age of 1 year. Our data showed a significant effect for age at time of surgery on later IQ, with SS and bicoronal craniosynostosis patients undergoing surgery at $\leq 6$ months having higher FSIQs and PIQs than those undergoing surgery later, and patients receiving surgery at $\geq 19$ months performing the least well.

It did, however, seem that the VIQ > PIQ discrepancy occurred regardless of whether patients had undergone surgery. Sixty-eight percent of the patients in this study had been operated on. Limited published data are available with which to compare this figure as the majority of papers on craniosynostosis focus on groups of patients who have undergone, or are due to undergo, surgical correction. In one study of patients with SS, 8 (44\%) of 18 cases had undergone surgery, ${ }^{9}$ and in another study, 63 (75\%) of 84 patients with single-suture craniosynostosis had been operated on. ${ }^{11}$ In our patient group, the apparently low number of patients electing to undergo surgery is, at least in part, because the developmental data were collected from every patient attending the service, and this will have included those with mild presentations. In our previous study (Bellew et al.5), we identified that 11 of 23 nonsurgical SS cases had had mild presentations. It is interesting, therefore, that the VIQ > PIQ discrepancy seems to occur regardless of whether surgery has taken place, as it suggests that the impact on development occurs even in mild cases.

\section{Sex}

The data on sex suggested that while males did not differ from females in terms of FSIQ, VIQ, or PIQ, they did differ from females in terms of showing the VIQ > PIQ pattern of development. This only reached significance when all types of craniosynostosis were combined and in the SS group, but the lack of significance with the other types of craniosynostosis may have reflected small sample size. However, it was striking that the female patients showed the opposite pattern (PIQ > VIQ) in about half of all cases, whereas the majority of male patients $(67 \%)$ showed VIQ > PIQ. Unfortunately, neither the Chieffo et al. ${ }^{7}$ or Magge et al. ${ }^{16}$ studies looked at the effects of sex, and so no comparison with the previous data can be made.

\section{Causal Basis}

Although there is now a growing body of evidence showing an increase in risk for cognitive deficits in patients with single-suture craniosynostosis, the causal basis for this remains unclear. There is a suggested inverse relationship between raised ICP and IQ, particularly in untreated cases, ${ }^{10,18,19}$ but the incidence has been shown to increase with the number of sutures involved, and it is generally accepted that raised ICP is unusual in single-suture craniosynostosis. It has been reported to occur in only $7 \%-13 \%$ of patients with $\mathrm{SS},{ }^{2}$ and in our previous study of developmental attainment in 5-year-old children with SS, ${ }^{5}$ only 1 child in a sample of 32 had confirmed raised ICP. In the current study, only 2 patients had confirmed raised ICP ( $>$ $20 \mathrm{~mm} \mathrm{HG}$ ). One had a diagnosis of pan craniosynostosis, and the other had SS and unilateral lambdoid craniosynostosis. It therefore seems unlikely that the current findings are related to raised ICP although this was not formally assessed in every case. All the patients, however, received regular developmental and ophthalmology reviews, to indirectly assess for raised ICP, with a low threshold to offer ICP monitoring to those parents not wanting to have surgery.

It is, of course, possible that there is a sample bias, as it could be hypothesized that children with problems are more likely to be brought to their follow-up appointments, and certainly by 10 years of age there is a large attrition rate. However, as most of the children had an IQ within the normal range, this seems unlikely to entirely account for the findings. Furthermore, it was striking that the numbers of patients who presented at 10 years were different proportionally to those who were operated on, or seen for developmental assessment, at less than 5 years of age. Typically, patient distribution is SS $>$ metopic $>$ unicoronal $>$ bicoronal $>$ multiple, but in the current sample there were fewer surgical metopic cases than would be expected. Traditionally these would be the patients expected to have more cognitive and behavioral problems ${ }^{6,21}$ and therefore more likely to turn up for assessment, but clearly this was not the case in the current study, and so bias cannot account for the research findings.

Kapp-Simon et al. ${ }^{14}$ note that anatomical studies of children without single-suture craniosynostosis have suggested that disruption to specific brain structures may be associated with particular cognitive deficits. It could, therefore, be hypothesized that an abnormally shaped skull, as present in craniosynostotic conditions, would produce a secondary deformation of the brain that could result in the disruption of normal neuropsychological development. Lin et al. ${ }^{15}$ conducted a comparative analysis of their "shape descriptors" in an attempt to understand the impact of skull deformations on neurobehavior. They found that scaphocephaly severity indices and symbolic shape signatures were predictive of mental ability and psychomotor functions, respectively, which suggests the possibility that secondary deformation could influence neurodevelopmental status. More recently, Beckett et al. ${ }^{3}$ have used 3-T MRI and diffusion tensor imaging to show altered neocortical structural and functional connectivity in SS, which they feel, in part, may explain the neuropsychological deficits commonly reported in this patient population.

\section{Conclusions}

The results of this study confirm the finding that at longterm follow-up, although children with nonsyndromic craniosynostosis fall within the normal range for intelligence, there is a VIQ > PIQ discrepancy above what would be expected in the normal population, and which may therefore be indicative of more subtle difficulties in achievement. 
This is also affected by type of craniosynostosis, sex, and age at time of surgery.

\section{Acknowledgments}

We would like to acknowledge the following clinical psychologists from the Leeds Teaching Hospitals NHS Trust, who all contributed to the data collection: Drs. Jane Clark, Chloe Miller, Andrea Brown, N. Wainwright, David Aaron, and Merry Hill. We would also like to acknowledge Mr. Mark Liddington (consultant plastic surgeon) and Mr. John Russell (consultant maxillofacial surgeon), members of the Leeds craniofacial surgical team.

\section{References}

1. Arnaud E: Discussion on American Society of Maxillofacial Surgeons Outcome Study: preoperative and postoperative neurodevelopmental findings in single-suture craniosynostosis. Plast Reconstr Surg 114:848-849, 2004

2. Arnaud E, Renier D, Marchac D: Prognosis for mental function in scaphocephaly. J Neurosurg 83:476-479, 1995

3. Beckett JS, Brooks ED, Lacadie C, Vander Wyk B, Jou RJ, Steinbacher DM, et al: Altered brain connectivity in sagittal craniosynostosis. J Neurosurg Pediatr 13:690-698, 2014

4. Bellew M, Chumas P, Mueller R, Liddington M, Russell J: Pre- and postoperative developmental attainment in sagittal synostosis. Arch Dis Child 90:346-350, 2005

5. Bellew M, Liddington M, Chumas P, Russell J: Preoperative and postoperative developmental attainment in sagittal synostosis: five-year follow-up. J Neurosurg Pediatr 7:121-126, 2011

6. Bottero L, Lajeunie E, Arnaud E, Marchac D, Renier D: Functional outcome after surgery for trigonocephaly. Plast Reconstr Surg 102:952-960, 1998

7. Chieffo D, Tamburrini G, Massimi L, Di Giovanni S, Giansanti C, Caldarelli M, et al: Long-term neuropsychological development in single-suture craniosynostosis treated early. J Neurosurg Pediatr 5:232-237, 2010

8. Di Rocco F, Knoll BI, Arnaud E, Blanot S, Meyer P, Cuttarree $\mathrm{H}$, et al: Scaphocephaly correction with retrocoronal and prelambdoid craniotomies (Renier's " $H$ " technique). Childs Nerv Syst 28:1327-1332, 2012

9. Hemple DJ, Harris LE, Svien HJ, Holman CB: Craniosynostosis involving the sagittal suture only: guilt by association? J Pediatr 58:342-355, 1961

10. Kaiser G: Sagittal synostosis-its clinical significance and the results of three different methods of craniectomy. Childs Nerv Syst 4:223-230, 1988

11. Kapp-Simon KA: Mental development and learning disorders in children with single suture craniosynostosis. Cleft Palate Craniofac J 35:197-203, 1998

12. Kapp-Simon KA, Figueroa A, Jocher CA, Schafer M: Longitudinal assessment of mental development in infants with nonsyndromic craniosynostosis with and without cranial release and reconstruction. Plast Reconstr Surg 92:831-841, 1993

13. Kapp-Simon KA, Leroux B, Cunningham M, Speltz ML: Multisite study of infants with single-suture craniosynostosis: preliminary report of presurgery development. Cleft Palate Craniofac J 42:377-384, 2005

14. Kapp-Simon KA, Speltz ML, Cunningham ML, Patel PK,
Tomita T: Neurodevelopment of children with single suture craniosynostosis: a review. Childs Nerv Syst 23:269-281, 2007

15. Lin HJ, Ruiz-Correa S, Shapiro LG, Speltz ML, Cunningham ML, Sze RW: Predicting neuropsychological development from skull imaging. Conf Proc IEEE Eng Med Biol Soc 1:3450-3455

16. Magge SN, Westerveld M, Pruzinsky T, Persing JA: Longterm neuropsychological effects of sagittal craniosynostosis on child development. J Craniofac Surg 13:99-104, 2002

17. Neumann CS, Walker EF: Childhood in neuromotor soft signs, behaviour problems, and adult psychopathology, in Ollendick TH, Prinz RJ (eds): Advances in Clinical Child Psychology. New York: Plenum Press, 1997

18. Renier D: Intracranial pressure in craniosynostosis: pre- and postoperative recordings - correlation with functional results, in Persing JA, Edgerton MT, Jane JA (eds): Scientific Foundations and Surgical Treatment of Craniosynostosis. Baltimore: Williams \& Wilkins, 1989, pp 263-269

19. Renier D, Sainte-Rose C, Marchac D, Hirsch JF: Intracranial pressure in craniostenosis. J Neurosurg 57:370-377, 1982

20. Shipster C, Hearst D, Somerville A, Stackhouse J, Hayward R, Wade A: Speech, language, and cognitive development in children with isolated sagittal synostosis. Dev Med Child Neurol 45:34-43, 2003

21. Sidoti EJ Jr, Marsh JL, Marty-Grames L, Noetzel MJ: Longterm studies of metopic synostosis: frequency of cognitive impairment and behavioral disturbances. Plast Reconstr Surg 97:276-281, 1996

22. Speltz ML, Kapp-Simon KA, Cunningham M, Marsh J, Dawson G: Single-suture craniosynostosis: a review of neurobehavioral research and theory. J Pediatr Psychol 29:651-668, 2004

23. Starr JR, Kapp-Simon KA, Cloonan YK, Collett BR, Cradock MM, Buono L, et al: Presurgical and postsurgical assessment of the neurodevelopment of infants with singlesuture craniosynostosis: comparison with controls. J Neurosurg 107 (2 Suppl):103-110, 2007

24. Wechsler D: Wechsler Abbreviated Scale of Intelligence (WASI). San Antonio, TX: Harcourt Assessment, 1999

25. Wechsler D: Wechsler Intelligence Scale for ChildrenThird Edition (WISC-III). San Antonio, TX: The Psychological Corporation, 1991

26. Wechsler D: Wechsler Intelligence Scale for ChildrenFourth UK Edition (WISC-IV UK). London: Pearson Assessment, 2004

\section{Author Contributions}

Conception and design: Bellew. Acquisition of data: Bellew. Analysis and interpretation of data: both authors. Drafting the article: Bellew. Critically revising the article: both authors . Reviewed submitted version of manuscript: both authors. Approved the final version of the manuscript on behalf of both authors: Bellew. Statistical analysis: Bellew.

\section{Correspondence}

Maggie Bellew, Department of Plastic, Reconstructive, and Hand Surgery, Leeds General Infirmary, Belmont Grove, Leeds LS2 9NS, United Kingdom. email: maggie.bellew@nhs.net. 